\title{
Acceso a las tic's en estudiantes afroecuatorianos en Santo Domingo de los Tsáchilas.
}

\author{
Access to tic 's in students Afro-ecuadorians in santo domingo de los \\ Tsáchilas.
}

Mg. Holger Arturo Intriago Mairongo. ${ }^{1}$, Dr. Fernando Lara Lara. ${ }^{2}$, Ing. Luz Irene Flores Abarca ${ }^{3}$, Ing. Rosa Virginia Cedeño Hugalde. ${ }^{4}$, Ing. Rentería Bolaños Luis Felipe. ${ }^{5}$

Recibido: 02-09-2017 / Revisado: 11-11-2017 Aceptado: 14-12-2017/ Publicado: 01-01-2018

\begin{abstract}
.
DOI: https://doi.org/10.33262/cienciadigital.v2i1.11

This article aims to describe some aspects that explain the difficulties of educational access to Information and Communication Technologies in the Afro - Ecuadorian students of seventh of Basic General Education of the Educational Unit "Brasilia". The problem for which the study is proposed is based on the digital exclusion experienced by Afro-Ecuadorian students of marginal urban educational institutions. The results of the study show that there is little access to Tics in the educational and scholastic context of students. In the conclusions, we describe aspects related to the low availability of technological resources in the educational context, in addition, explains the lack of access to the internet and a computer in the students' homes, which possibly affects the use and knowledge of certain tools technologies.
\end{abstract}

Keywords: Education, Technology, Information Technology, Social Exclusion

\section{Resumen.}

El presente artículo se plantea con el objetivo de describir algunos aspectos puedan evaluar de manera aproximativa las dificultades de acceso educativo a las Tecnologías

\footnotetext{
1 Universidad Luis Vargas Torres, Esmeraldas, Ecuador, holger.intriago@utelvte.edu.ece 2 Pontifica Universidad Católica, Santo Domingo, Ecuador, llf@pucesd.edu.ec 3 Universidad Luis Vargas Torres, Esmeraldas, Ecuador, flor.silvestre.007@hotmail.com 4 Universidad Luis Vargas Torres, Esmeraldas, Ecuador, jomyke2013@outlook.com 5 Universidad Luis Vargas Torres, Esmeraldas, Ecuador, luisfelipe_7319@hotmail.com
} 
de la información y comunicación en los estudiantes afroecuatorianos de séptimo de Educación General Básica de la Unidad Educativa "Brasilia". El problema por el cual se propone el estudio se asienta en la exclusión digital que experimentan los estudiantes afroecuatorianos de instituciones educativas urbano marginales. Los resultados del estudio vienen a comprobar que existe un escaso acceso a las Tics en el contexto educativo y escolar de los estudiantes. Del mismo modo, aunque se han implementado políticas públicas de conexión digital, parecen no haberse evaluado correctamente. En las conclusiones, se describen aspectos relacionados a la poca disponibilidad de recursos tecnológicos en el contexto educativo, además, se pone en evidencia la carencia de acceso a internet y un ordenador en los hogares de los estudiantes evidenciándose la necesidad de complejizar la visión a los distintos contextos educativos.

Palabras Claves: Educación, tecnología, TIC, exclusión social.

\section{Introducción.}

Los afrodescendientes han sido excluidos durante muchos momentos de la historia (especialmente en el Esclavismo), ya que no se los consideraba seres humanos, sino por el contrario, eran utilizados como esclavos, además de ser tratados inhumanamente. Se podría detenerse en la evolución del concepto de persona, que aunque no sea cuestión de este trabajo sí forma parte del debate acerca de si existe conciencia con relación al significado contenido en las distintas normativas nacionales e internacionales que enmarcan nuestras democracias. Así, junto con otros colectivos de atención preferente parece ser un hecho los actos excluyentes hacia los afrodescendientes, en especial cuando se evidencia actos de racismo. De acuerdo a esto, Sánchez (2011) considera que:

...La mayoría de los afrodescendientes en las Américas, comparten una realidad social caracterizada por el racismo estructural. Este tipo de racismo incubado desde las estructuras coloniales y esclavistas, ha repercutido en factores adversos al desarrollo y a la satisfacción de los derechos humanos en los afrodescendientes. Se trata de la pobreza, la desigualdad y la exclusión social. (p.76) 
La pobreza, la desigualdad, la exclusión y el racismo que experimentan los afrodescendientes no solo se evidencia es aspectos culturales y sociales, sino también, en el ámbito educativo (Constitución de la República del Ecuador, 2008; Ley Orgánica de Educación Intercultural, 2011). Los afrodescendientes históricamente han sufrido la desigualdad en el acceso a la educación, pues en un principio, no formaban parte de la estructura social; por otro lado, era más complicado para este colectivo acudir al blanqueamiento que explica Enrique Ayala Mora por razones físicas, o quizás por el propio orgullo identitario. La desigualdad social de los afros supone su consecuente distanciamiento en su formación educativa, contribuyendo a la polarización como colectivo desfavorecido traducido en altos niveles de pobreza y exclusión social en esta población (Vicariato Apostólico de Esmeraldas \& Centro Cultural Afroecuatoriano, 2009), retroalimentando su condición educativa desfavorecida y el rol que Aníbal Quijano denomina de colonialidad del poder.

En América y otros continentes todavía se evidencian algunos rezagos de racismo y exclusión social de los afrodescendientes. Esta etnia sufre numerosos problemas socio-económicos que le impiden su desarrollo, consistentes en una vida precaria y con un salario mínimo; esto significa que estas personas sufran de pobreza (en algunos casos de indigencia), alejados del acceso a servicios básicos y mejores condiciones de vida. En este sentido se pronuncia la UNICEF (2016) al considerar que:

La pobreza como manifestación de la exclusión social se reproduce con mayor intensidad en la población afrodescendiente y en otras minorías étnicas, ya que, además de los problemas y las privaciones que se derivan de la situación de pobreza, estos grupos deben enfrentar obstáculos adicionales como resultado de los rezagos históricos y la discriminación. (p.26)

En cuanto a Ecuador se refiere, objeto de este trabajo se enmarca en el desafío educativo que se plantea a través de su ordenamiento jurídico y sus políticas públicas, pues consiste en nuestra opinión fundamentalmente en un desafío en torno a la inclusión educativa, concretamente en el caso de los afroecuatorianos (Córdoba, Lara, \& García, 2017; Constitución de la República del Ecuador, 2008; Ley Orgánica de Educación Intercultural, 2011; SENPLADES, 2013; Lara, 2015; Lara \& Herrán, 2016; Lara, et al, 2016). En este 
sentido, la compleja problemática que se presenta hace necesaria su acotación para buscar la practicidad, en nuestro caso es la dimensión educativa que se ocupa de la exclusión digital, en especial los niños, niñas y adolescentes afroecuatorianos. La relevancia de su detenimiento se constata en que los actuales sistemas educativos se orientan a la inserción de las tecnologías de la información y la comunicación en el aula de clases, pero la brecha digital que se establece entre los sectores educativos centrales y marginales, marca diferencias con respecto al acceso a las Tecnologías de la información y la comunicación. Esta preocupación en Ecuador se torna en un derecho animado a erradicar el analfabetismo digital (Constitución de la República del Ecuador, 2008) desde el enfoque de la inclusión educativa (Córdoba, Lara, \& García, 2017). Sin embargo, parecen desconocerse las consecuencias que han tenido las políticas educativas del buen vivir en este colectivo, y del mismo modo en la erradicación de un analfabetismo que se comprende ya desde lo digital (Lara \& Herrán, 2016)

Sobre la base de lo expuesto, Calvo \& Rojas (2007) consideran que la educación debe favorecer el acceso a las Tics a todas las personas y en especial a las que presentan algún tipo de exclusión, ya que "nos encontramos ante personas que quedan, en alguna medida, al margen de estos procesos de participación, dando lugar a dinámicas de exclusión digital y social" (p.145). En el Ecuador no es la excepción, con diferencias sociales, discriminación, exclusión social y tecnológica en la población afroecuatoriana entre otras. Esta realidad se constata en estudios realizados por el Programa de las Naciones Unidas para el Desarrollo (2010) que demuestran que los afroecuatorianos y en general los afrodescendientes tienen peores condiciones de vida y situaciones de exclusión que las poblaciones pertenecientes a otras etnias. Así, de forma general los afrodescendientes tienen problemas socio-económicos y educativos que los convierte en una población excluida o en algunos casos desfavorecida de servicios básicos para un adecuado nivel de vida. Siendo la educación una tarea de necesario tratamiento por cuanto la exclusión educativa es una realidad, así como también la exclusión digital en el proceso de enseñanza aprendizaje. Se concibe en este trabajo que la exclusión digital provoca dificultades para la alfabetización mediática en este colectivo; ahora bien ¿la accesibilidad va ligada a la formación? ¿La escuela es un contexto importante de alfabetización mediática en este colectivo o tienen mayor peso otros? 
Con la señalado en líneas anteriores, el presente estudio persigue el acercamiento a la realidad educativa de unidades escolares integradas por población afrodescendiente para contrastar los esfuerzos de las políticas públicas para la alfabetización mediática en estos colectivos con la cotidianidad presente, así como intentar realizar un esfuerzo por visibilizar las carencias de este colectivo y fomentar una cultura educativa favorecedora de un enfoque inclusivo de la formación. Si bien los datos no son representativos, entendemos que pueden servir para orientar futuras investigaciones. Entendemos que la enculturación propiamente del buen vivir de la comunidad educativa pasa por la accesibilidad a las TICs por cuanto la complejidad de la realidad educativa actual demanda de "estar a la altura de los tiempos" como nos recordaba Ortega; en nuestro caso el entendimiento de la realidad educativa cada vez más globalizada es presidida por nuevos significados (Lara \& Gerstner, 2016) desde las posibilidades y riesgos de las nuevas tecnologías, pues en numerosos casos no se "sabe" utilizar o no se "puede acceder".

\section{Materiales y métodos.}

El diseño seguido para la investigación realizada fue no experimental, pues según Hernández, Fernández \& Baptista (2010) son "estudios que se realizan sin la manipulación deliberada de variables y en los que solo se observan los fenómenos en su ambiente natural para después analizarlos" (p.149). Responde a un trabajo cuantitativo de tipo descriptivo en el que se aplicó la encuesta como instrumento de recogida de datos debidamente validado por expertos a docentes y estudiantes. Los datos fueron analizados estadísticamente a través del programa informático Excel.

La investigación es descriptiva, ya que esta "busca especificar propiedades, características y rasgos importantes de cualquier fenómeno que se analice. Describe tendencias de un grupo o población" (Hernández, Fernández \& Baptista, 2010, p.80). De acuerdo a esto se persigue conocer los hechos que explican las dificultades de acceso educativo de las Tecnologías de la información y comunicación con respecto a los estudiantes afroecuatorianos de séptimo de Educación General Básica y los docentes de la unidad educativa antes mencionada. 
La muestra fue de tipo intencional, comprende a 7 docentes y 27 estudiantes afroecuatorianos de la Unidad Educativa "Brasilia". Ubicada en las riveras del Rio Toachi a la cual asisten estudiantes que se encuentran en los asentamientos con personas afrodescendientes.

\section{Resultados.}

TABla 1. Acceso y CONOCIMIENTO de LAS tICS De estudiantes y DOCEnTES.

\begin{tabular}{lc}
\hline \multicolumn{1}{c}{ Asignatura de computación dentro de horario de clases } & $\mathbf{4 \%}$ \\
\hline Acceso a laboratorio de computación en la escuela & $11 \%$ \\
Uso necesario de internet para hacer deberes & $93 \%$ \\
Existencia de computadora en casa & $22 \%$ \\
Internet en el hogar & $44 \%$ \\
Centro de cómputo cerca de casa & $74 \%$ \\
Uso del internet para tareas y chatear en redes sociales & $74 \%$ \\
Importancia del internet en la educación & $100 \%$ \\
Uso de correo electrónico & $0 \%$ \\
Conocimiento y uso de plataformas virtuales & $0 \%$ \\
Conocimiento de docentes sobre lo que son las tecnologías de la información y comunicación & $100 \%$ \\
Las docentes conocen los procesadores de texto, correo electrónico y las páginas web. & $55 \%$ \\
Curso de formación relacionado con las TICS & $86 \%$ \\
\hline
\end{tabular}

Elaborado por: Grupo de Investigación

De acuerdo con los resultados, el 4\% de los estudiantes afroecuatorianos indican que han recibido la asignatura de computación dentro de su horario de clases. En paralelo con el resultado descrito, el $11 \%$ de los estudiantes indican que han accedido al laboratorio de computación (ver tabla 1). A partir de los porcentajes expuestos, se plantea una primera aproximación al problema de acceso a las Tics que tienen los estudiantes afroecuatorianos en la institución.

Con relación a los resultados, se demuestra que el 93\% de los estudiantes necesitan internet para hacer deberes, sin embargo, solo el $44 \%$ de los encuestados tienen acceso a internet en sus hogares; de la misma manera, el 22\% de los estudiantes indican que tienen un computador en sus casas (ver tabla 1). Con respecto a la necesidad de un computador y acceso a internet en el hogar, el $74 \%$ de estudiantes indican que tienen un centro de cómputo cerca de su casa, 
mediante el cual, el mismo porcentaje lo utilizaría para acceder a internet y chatear en redes sociales (ver tabla 1$)$.

Sobre la base de los resultados, el 100\% de los estudiantes señalan que el internet es de suma importante para su educación (ver tabla 1); sin embargo, se viene a comprobar que ninguno de los estudiantes usa correo electrónico, de la misma manera, ninguno de los estudiantes conoce o usa plataformas virtuales de aprendizajes. Por lo tanto, parece ser que el problema de acceso y conocimiento de las Tics se está acentuando en el contexto educativo y familiar de los estudiantes afroecuatorianos encuestados.

Es importe reconocer, que las docentes de esta Unidad Educativa afirman en su totalidad tener conocimientos sobre las tecnologías de la información. E1 55\% de las docentes conocen los procesadores de texto, correo electrónico y las páginas web además el $86 \%$ de las docentes dicen haber recibido un curso de formación relacionado con las tecnologías de la información y comunicación.

\section{Discusión.}

Los resultados de la investigación vienen a comprobar que los estudiantes afroecuatorianos parecen estar siendo excluidos del acceso a la tecnología, ya que, la institución no tiene un laboratorio de computación y un servicio adecuado de internet. Esta arista de exclusión que experimentan implícitamente estos estudiantes, tiene relación con el planteamiento de Sánchez (2011), el cual considera que los afrodescendientes comparten una realidad social similar, que tiene vinculación con la pobreza, desigualdad y la exclusión social, dando como resultado, un escaso acceso a las Tics en el contextos escolar, familiar y comunitario.

La exclusión social y tecnológica de los estudiantes afroecuatorianos de la institución, posiblemente repercuta su formación educativa, que se según el Vicariato Apostólico de Esmeraldas \& Centro Cultural Afroecuatoriano (2009), dan como resultado altos niveles de pobreza y exclusión social en esta población. El contexto educativo y realidad social de los estudiantes afrodescendientes es una manifestación de la exclusión de minorías étnicas, que pueden ser obstáculos adicionales relacionados a los rezagos históricos y la discriminación de los afrodescendientes (UNICEF, 2016). 
La falta de un laboratorio de computación y un adecuado acceso a internet en la institución, imposibilita que los estudiantes afrodescendientes reciban la asignatura de computación, además, la gran mayoría de docentes no utilizan computadora para enseñar algún tema de clase. Sobre la base de lo expuesto, parece ser que la falta de recursos tecnológicos y la nula o escasa iniciativa de aplicación de las Tics en el aula de clases, provoca que los estudiantes afroecuatorianos queden al margen de este proceso participativo, y de acuerdo a Calvo \& Rojas (2007), esto puede dar lugar a la exclusión social y el incremento de la brecha digital.

La repercusión de la exclusión digital y el posible incremento de la brecha digital que parecen tener los estudiantes afroecuatorianos de la institución, puede ser en un momento determinado, un factor de exclusión laboral para este grupo étnico, ya que, según la Comisión Económica para América Latina y el Caribe (2002) cada vez más, el peso de la educación determina el acceso a mejores puestos de trabajo e ingresos económicos más altos. Por lo tanto, es necesario tomar conciencia de la importancia del uso de las Tics en procesos educativos, en razón de que, disminuyen la brecha digital y la exclusión digital de grupos étnicos minoritarios, como es el caso de los afroecuatorianos.

De acuerdo a los resultados, el mayor porcentaje de estudiantes afroecuatorianos tiene que utilizar necesariamente internet para realizar sus tareas, aun así, la mayor parte de ellos no poseen una computadora en su hogar. Este resultado tiene relación con lo planteado en el Programa de las Naciones Unidas para el Desarrollo (2010), en el cual, se considera a la población afroecuatoriana como un grupo étnico experimenta deferencias sociales, desigualdad y discriminación.

El limitado acceso a internet y disponibilidad de una computadora por parte de los estudiantes afroecuatorianos de la institución, evidencia el posible incumplimiento de políticas de inclusión digital que se establecen a nivel mundial y nacional, como es el caso de la Conferencia Mundial contra el Racimos, la Discriminación Racial, la Xenofobia y las Formas Conexas de Intolerancia y el artículo 347, numeral ocho de la Constitución de la República del Ecuador (2008), que instan al Estado a que se garantice el acceso a la educación y promuevan el acceso a las nuevas tecnologías (Asamblea Nacional del Ecuador, 2008), de 
modo que los afrodescendientes tengan los recursos necesarios para la educación, desarrollo tecnológico y el aprendizaje (Comisión Económica para América Latina y el Caribe, 2009).

Los estudiantes afrodescendientes que no tienen computadora o internet en casa, deben acudir a centros de cómputo de su localidad, ya que, necesitan internet entre dos, cinco o más veces a la semana para hacer tareas, pero también, para interactuar atreves de redes sociales. De acuerdo al Programa de las Naciones Unidas para el Desarrollo (2010) los afrodescendiente se encuentran en una situación desfavorable en lo que respecta a la educación, por esta misma razón, la dificultad de acceso a la tecnología y a internet, que experimentan los estudiantes afrodescendientes de la institución educativa mitiga un adecuado proceso de enseñanza y aprendizaje, en el que puedan desarrollar competencias en el uso y aprendizaje por medio de las tecnologías.

Con relación a los resultados obtenidos y sobre la base del planteamiento de Antón (2005) se podría mencionar que los afroecuatorianos son el segundo grupo étnico con ingresos más bajos por habitante, además de tener tasas bajas de asistencia escolar, secundaria y universitaria. Estos aspectos económicos y sociales se reflejan en la educación de los estudiantes afroecuatorianos de la institución, ya que, se deben movilizar fuera de sus hogares para tener acceso a las tecnologías.

Por otro lado, los resultados vienen a comprobar que en la institución educativa no pueden acceder a internet, y de acuerdo a la Secretaría Nacional de Planificación y Desarrollo (2013), en el plan Nacional del Buen Vivir 2013-2017, se estableció como meta hasta el 2017 equipar con internet al $90 \%$ de las instituciones educativas. Debido a la carencia de acceso a internet en la institución, parece ser que la unidad educativa estuvo fuera del $90 \%$ de las instituciones educativas equipadas con internet, por esta razón, pareciera que la exclusión social, digital y laboral siguen siendo factores adversos para los afroecuatorianos, en razón de que, Halvorsen \& Vale (2012) consideran que son el impedimento para un acceso masivo, de calidad e inclusivo a las instituciones educativas. Sin embargo, sí se puede constatar que ha existido una inversión pública de dotación de servicio de acceso a internet, situación que no está enmarcado dentro de las políticas públicas. 
En relación con los resultados, los estudiantes afroecuatorianos consideran importante el uso de tecnologías en su educación, ya que, ninguno utiliza correo electrónico, plataformas virtuales, redes sociales u otro medio tecnológico para hacer tareas o aprender. La conciencia de la importancia de las Tics en los estudiantes afroecuatorianos se enlaza con la omnipresencia de las tecnologías digitales en las nuevas generaciones, que en definitiva son necesarias en los procesos educativos y el desarrollo de destrezas cognitivas (UNESCO, 2013).

La apertura tecnológica de las nuevas generaciones afroecuatorianas, plantean posibilidades ilimitadas en su proceso educativo, ya que, por medio de la Tics se puede dinamizar el conocimiento, expandirlo y hacerlo accesible para las personas (Zhang, Yang, Chang \& Chang, 2016). Por esta razón, surge la necesidad de enfrentar la exclusión digital y disminuir la brecha digital de los afroecuatorianos, en especial, de las nuevas generaciones.

Los resultados vienen a comprobar que los docentes conocen sobre los tipos de Tics que se pueden aplicar en la educación, debido a su formación profesional de tercer nivel, por lo tanto, tienen la capacidad de aplicarlas en su planificación. Además, poseen una computadora $y$ acceso a internet en el hogar, que son utilizados frecuentemente para diferentes actividades profesiones.

El conocimiento de las Tics y la posibilidad de acceso, que tienen los docentes de la institución puede ser un aliciente para la aplicación de tecnologías en el aula de clases, que de acuerdo a Alcántara (2009) se convierten en un método o estrategia que promueve el aprendizaje de los estudiantes. Además, Moreno (2011) menciona que las Tics y la multimedia se han convertido en recursos idóneos para los enfoques educativos centrados en el alumno, por lo tanto, existe la posibilidad de mejorar la praxis docente en la institución, por medio del uso de recursos tecnológicos.

A pesar de que los docentes conocen sobre las Tics, no las aplican en el aula de clases, porque no disponen de computadoras o de un laboratorio de computación con acceso a internet en la institución. Esta realidad del contexto escolar de los docentes y estudiantes afroecuatorianos, afecta el proceso educativo. Sobre la base de lo explicado, parece ser que la escasa presencia de las tecnologías puede relacionarse con la falta de innovación educativa de los docentes y 
autoridades de la institución (Boza \& Toscano, 2011). Por esta razón, la falta de recursos tecnológicos en la unidad educativa, limita la iniciativa de los docentes de la institución por aplicar las Tics en el aula de clases, que de una u otra manera, es factor para la permanencia de la exclusión digital de los estudiantes, y en especial de los estudiantes afroecuatorianos.

La exclusión digital de los estudiantes afroecuatorianos de la institución, no solo se determina en el contexto escolar, sino también en el contexto social y familiar, porque la institución no dispone de los recursos tecnológicos para implementar las Tics en el proceso de enseñanza y aprendizaje, por lo tanto, esta carencia de recursos se contrapone a lo planteado García \& Tejedor (citado por Boza \& Toscano, 2011), los cuales menciona la importancia de la disponibilidad de dotación suficiente de tecnología en las instituciones educativas, en atención a las necesidades y diversidad de los estudiantes.

Sobre la base de lo explicado, se establece que las necesidades tecnológicas de la institución, están repercutiendo la educación de los estudiantes afroecuatorianos y la innovación de los docentes, ya que, aún no se están aplicando la potencialidades de las Tics como herramientas de ayuda dentro del proceso educativo, asimismo, la posibilidad de acceso a la mayor cantidad de estudiantes (Martínez, Pérez \& Martínez, 2015).

De acuerdo a los resultados, existe una nula aplicación de las Tics para impartir clases o mejorar el proceso de enseñanza y aprendizaje, esto posiblemente incrementa la exclusión tecnológica de los estudiantes de la institución, y mucho más a lo que pertenecen a la etnia afrodescendiente. Esta problemática identificada en la institución, muestra la inconsistencia de ciertas políticas de inclusión digital en el Ecuador, ya que, no se está dando cumplimiento; el artículo 347, numeral 7 de Constitución del Ecuador (2008) que hace referencia a la erradicación del analfabetismo puro, funcional y digital, para así superar el rezago educativo. En el mismo sentido, tampoco se está asegurando el acceso universal a las tecnologías de la información y comunicación considerado en la Constitución del Ecuador (2008).

Al parecer, las políticas públicas sobre inclusión digital aún no son una realidad en la institución educativa, por lo tanto, se puede establecer que existe una inconsistencia con el artículo 2, literal v, de la Ley Orgánica de Educación Intercultural, que considera la equidad e inclusión permanente en el sistema educativo, además garantizar la igualdad de 
oportunidades a comunidades, pueblos, nacionalidades y grupo con necesidades educativas especiales. Sobre la base de esta perspectiva, parece ser que la exclusión educativa y en especial la exclusión digital, mantengan la latente la tasa de $8,66 \%$ de analfabetismo de los afroecuatorianos (Defensoría del Pueblo del Ecuador, 2012).

En definitiva, es necesaria que las políticas de inclusión digital sean ejecutadas procedimentalmente en la institución (recursos tecnológicos y acceso a internet), para mitigar sistemáticamente la brecha digital que pueden tener los estudiantes afroecuatorianos en la unidad educativa, además de posibilitar la innovación tecnológica que los docentes pueden llevar a cabo por medio de las Tics. Es entonces relevante, tomar a consideración la Declaración del Milenio (citado por Farfán, Medina \& Caheiro, 2015), la cual enfatiza la necesidad de incorporarlas Tics en la educación como un elemento estratégico para la inclusión digital de la sociedad.

A más de la necesidad imperiosa de aplicar las políticas educativas ecuatorianas en materia de Tics, es importante que se evalúe su aplicación, ya que, de no ser así se puede estar sectorizando las políticas sobre inclusión digital, dando como resultado una inconsistencia en la promulgación de un acceso inclusivo a las Tics en todos las instituciones educativas. Sobre este problema Lugo \& Kelly (2010) consideran necesario que el Estado se comprometa en la masificación de las Tics sin sectorizar su aplicación; además se debe tomar en cuenta que la evaluación de estas políticas debería ser pública y con la finalidad de mejorar los fundamentos curriculares, axiológicos, didácticos, pedagógicos, sociales, culturales y étnicos vigentes en el sistema educativo ecuatoriano (Calero \& Travitzki, 2014).

\section{Conclusiones.}

- Los estudiantes afroecuatorianos parecen estar siendo excluidos del acceso a la tecnología, debido a que, la institución no tiene un laboratorio de computación y un servicio adecuado de internet, por lo tanto, no reciben la asignatura de computación. De la misma manera, los resultados demuestran que los estudiantes utilizan necesariamente internet para realizar las tareas que se envía para la casa, aun así, se evidencia que la mayor parte de estos estudiantes no poseen un computador, y tan solo la mitad de ellos tienen acceso a internet en su hogar; provocando que deban 
movilizarse a centros de cómputo que quedan en su localidad para hacer sus tareas o para interactuar en redes sociales.

- Los estudiantes afroecuatorianos consideran importante el uso de las Tics en su proceso educativo, ya que, ninguno conoce o utiliza correo electrónico, plataformas virtuales u otros recursos tecnológicos para hacer tareas o aprender autónomamente. La nula aplicación de las Tics para impartir clases o mejorar el proceso de enseñanza y aprendizaje, incrementa la exclusión tecnológica de los estudiantes de la institución, y mucho más a lo que pertenecen a la etnia Afrodescendiente.

- Con base a lo mencionado, parece ser que la brecha digital que están experimentando los estudiantes afroecuatorianos de la institución, comprueba que las políticas de acceso a las tecnologías del Ecuador aún no se están cumpliendo en todas las instituciones educativas. Por esta misma razón, la evaluación de las políticas públicas de inclusión digital en la educación, es un elemento trascendental en la mejora de los elementos del sistema educativo ecuatoriano, ya que, de no ser así, se puede sectorizar el acceso a las Tics, afectando a grupos humanos vulnerables y minoritarios, como el caso de los afroecuatorianos.

\section{Referencias bibliográficas.}

Alcántara, D. (2009). Importancia de las Tic para la educación. Recuperado (16/04/2017) de http://www.csicsif.es/andalucia/modules/mod_ense/revista/pdf/Numero_15/MARIA\%20DOLORE S_ALCANTARA_1.pdf

Antón, J. (2005). Los afroecuatorianos en cifras. Recuperado (10/04/2017) de http://www.siise.gob.ec/siiseweb/PageWebs/pubsii/pubsii_0052.pdf

Antón, J. (2005). Sistema de indicadores sociales del pueblo Afroecuatoriano. Recuperado (27/03/2017) de http://www.cepal.org/mujer/noticias/noticias/5/27905/JAnthon.pdf

Asamblea Nacional del Ecuador (2008). Constitución de la República del Ecuador. Recuperado(25/03/2017)http://www.asambleanacional.gov.ec/documentos/constituc ion_de_bolsillo.pdf 
Boza, A. \& Toscano, M. (2011). Buenas prácticas en integración de las TIC en educación en Andalucía: Dos estudios de caso. Recuperado (03/04/2017 de https://www.uv.es/aidipe/congresos/Ponencia_VIICongresoVirtual_AIDIPE.pdf

Calero, J. \& Travitzki, R. (2014). Evaluaciones de valor añadido en el ámbito educativo. Revista de Evaluación de Programas y Políticas Públicas, 1(2), 55-77. Doi: http://dx.doi.org/10.5944/reppp

Calvo, A. \& Rojas, S. (2007). Exclusión social y tecnológica. Comunicar, 15(29), 143-148. Recuperado(06/04/2017)dehttp://www.revistacomunicar.com/verpdf.php?numero=2 9\&articulo $=29-2007-23$

Comisión Económica para América Latina y el Caribe (2002). La equidad y la inclusión de los pueblos indígenas y afrodescendientes en América Latina y el Caribe. Revista de la CEPAL, 76(1), 39-54. Recuperado de https://www.academia.edu/485632/La_equidad_y_exclusi\%C3\%B3n_de_los_puebl o_ind\%C3\%ADgenas_y_afrodescendientes_en_Am\%C3\%A9rica_Latina_y_el_Car ibe

Comisión Económica para América Latina y el Caribe (2009). Afrodescendientes en América Latina y el Caribe: del reconocimiento estadístico a la realización de derechos. Recuperado (17/04/2017) de http://www.cinu.mx/minisitio/Afrodescendientes/Afrodescendientes.pdf

Constitución de la República del Ecuador. (2008). Ciudad Alfaro: Asamblea Constituyente. Córdoba, E.F., Lara, F. \& García, A. (2017). El juego como estrategia lúdica para la educación inclusiva del buen vivir. Ensayos, 32(1). 81-92. doi: 10.18239/ensayos.v32i1.1346

Defensoría del Pueblo del Ecuador (2012). El pueblo afrodescendiente en el Ecuador. Recuperado (18/04/2017) de http://www.corteidh.or.cr/tablas/28697.pdf

Farfán, S., Medina, A. \& Caheiro, M. (2015). La inclusión digital en la educación de Tarija, Bolivia. Revista CEPAL, 115(1), 71-90. Recuperado (07/03/2017) de http://repositorio.cepal.org/bitstream/handle/11362/37831/RVE115Farfan_es.pdf?se quence $=1$

Halvorsen, T. \& Vale, P. (2012). One World, many Knowledges: Reginal experiences and cross-regional links in higher education. Recuperado (06/03/2017) de 
https://books.google.com.ec/books?hl=es\&lr=\&id=J0wxDAAAQBAJ\&oi=fnd\&pg $=$ PA71\&dq=+educational+exclusion+in+black + people\&ots=LOrENOApQH\&sig= XPfCpCjHAh6JvupD7GuID4PKZYY\#v=onepage\&q\&f=false

Hernández, R., Fernández, C. \& Baptista, M. (2010). Metodología de la investigación. México: McGRAW-HILL

Lara, F. (2015). Personalismo en la legislación educativa ecuatoriana: una aproximación desde el pensamiento de Gabriel Marcel. Academia, (Número Especial: III Congreso Internacional de la Asociación Iberoamericana de Personalismo, LojaEcuador), 230-238. Recuperado de http://www.saber.ula.ve/bitstream/123456789/41273/3/articulo24.pdf

Lara, F., \& Gerstner, R. (2016). Inmigración y el Buen Vivir en Youtube: una experiencia en clase de ética. Trances, 8(3), 203-222. Recuperado de: http://www.trances.es/papers/TCS\%2008_3_3.pdf

Lara, F., Mena, P. R., \& Zambrano, M. J. (2016). “Aprendiendo a vivir mejor”: proyecto de educación inclusiva de La Pontificia Universidad Católica del Ecuador Sede Santo Domingo. Trances, 8(4):223 -246. Recuperado de http://www.trances.es/papers/TCS\%2008_4_1.pdf

Lara, F., Sousa, C., De la Herrán, A., Lara-Nieto, M.C., y Gerstner, R. (2016). El docente inmigrante "irregular" en Ecuador: reto del derecho a la Educación. Conhecimiento y Diversidade, 8(16), 25-43. doi:10.18316/rcd.v8i16.3362

Lara, F., y Herrán, A. (2016). Reflexiones sobre la educación del sumak kawsay en Ecuador. Revista Araucaria, 18(36), 41-58. Recuperado de https://goo.gl/TSF9ic Ley Orgánica de Educación Intercultural. (2011). Quito: Asamblea Constituyente.

Lugo, M. \& Kelly, V. (2010). Tecnología en educación ¿Políticas para la innovación? Recuperado(07/03/2017)http://www.udelas.ac.pa/biblioteca/librospdf/Documento T ecnologia_en_educ_Lugo_Kelly.pdf

Martínez, P., Pérez, J. \& Martínez, M. (2016). Las Tics y el entorno virtual para la tutoría universitaria. Educación XXI, 19(1), 287-310. doi: 10.5944/educXX1.13942

Ministerio de Educación del Ecuador (2007). Plan Decenal de Educación 2006-2015. Recuperado(03/04/2017)http://tbinternet.ohchr.org/Treaties/CEDAW/Shared\%20D ocuments/ECU/INT_CEDAW_ARL_ECU_18971_S.pdf 
Moreno, F. (2011). La multimedia como herramienta para el aprendizaje autónomo del vocabulario del inglés por parte de los niños. Colombian Applied Linguistics Journal,13(1),8494.Recuperado(06/03/2017)http://www.redalyc.org/articulo.oa?id= 305726660006

Programa de las Naciones Unidas para el Desarrollo (2010). Situación socioeconómica de la población afroecuatoriana en el marco de los Objetivos de Desarrollo del Milenio. Recuperado(24/04/2017)http:/www.afrodescendientesundp.org/FCKeditor_files/Fil e/AFRO_ECUADOR.pdf

Registro Oficial No. T.4691-SNJ-11-195 del 10 de febrero (2011). Ley Orgánica de Educación Intercultural. Recuperado (28/04/2017) de http://diccionario.administracionpublica.gob.ec/adjuntos/2loei.pdf

Riascos, S., Quintero, D. \& Ávila, P. (2009). Las TIC en el aula: percepciones de los profesores universitarios. Educación y educadores, 12(3), 133-157. Recuperado (21/04/2017)http://educacionyeducadores.unisabana.edu.co/index.php/eye/article/vi ew/1536/1841

Sánchez, A. (2011). El proceso organizativo afroecuatoriano: 1979-2009. Recuperado (14/03/2017) de http://www.flacsoandes.edu.ec/libros/digital/52015.pdf

Secretaría Nacional de Planeación y Desarrollo (2013). Objetivos nacionales del Buen Vivir. Recuperado (28/04/2017) de http://www.buenvivir.gob.ec/objetivo-2.auspiciar-la-igualdad-la-cohesion-la-inclusion-y-la-equidad-social-y-territorial-enla-diversidad

Secretaría Nacional de Planificación y Desarrollo (2015). Agenda Zonal 4. Recuperado (26/04/2017) de https://www.youtube.com/watch?v=Nck6BZga7TQ SENPLADES. (2013). Plan Nacional para el Buen Vivir 2013-2017. Quito, Ecuador. UNESCO (2008). Educación Inclusiva, El Camino hacia el Futuro, Reunión 48 de laConferenciaInternacionaldeeducación.Recuperado(17/03/2017)http://www.ibe.une sco.org/en/resources?search_api_views_fulltext $=\% 22$ Policy $\% 20$ Dialogue $\% 2048$ th

UNESCO (2008). La educación inclusiva: El camino hacia el futuro. Recuperado (25/04/2017)http://www.ibe.unesco.org/fileadmin/user_upload/Policy_Dialogue/48t h_ICE/CONFINTED_48_Inf_2_Spanish.pdf 
UNESCO (2013). Enfoques estratégicos sobre las Tics en educación en América Latina y el Caribe.Recuperado(24/03/2017)http://www.unesco.org/new/fileadmin/MULTIMED IA/FIELD/Santiago/images/ticsesp.pdf

UNICEF Ecuador (2016). El saber afroecuatoriano es parte de la identidad ecuatoriana, se presentó la Enciclopedia del Saber Afroecuatoriano. Recuperado (20/03/2017) de http://www.unicef.org/ecuador/media_15260.htm

Vicariato Apostólico de Esmeraldas \& Centro Cultural Afroecuatoriano (2009).

Enciclopedia del saber afroecuatoriano. Quito: Gráficas Iberia.

Zhang, J., Yang, J., Chang, M. \& Chang, T. (2016). ICT in Education in Global Context: The best practices in K-12 Schools. Recuperado (20/02/2017) de https://books.google.com.ec/books?hl=es\&lr=\&id=YsmbCwAAQBAJ\&oi=fnd\&pg $=$ PR5\&dq $=+$ Use + of $+\mathrm{ICT}+$ in + education\&ots $=U$ wFfj3fXho\&sig $=Y U C 13 p Y j b 7 \mathrm{VbB}$ Vw5tF0_zizTpQ0\#v=onepage\&q=Use $\% 20$ of $\% 20 I C T \% 20 \mathrm{in} \% 20$ education $\& \mathrm{f}=$ false

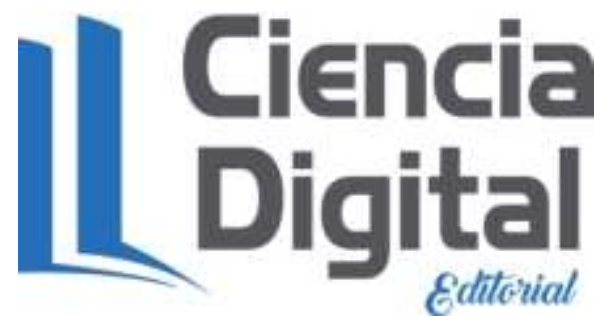




\section{Para citar el artículo indexado.}

Intriago H., Larra F., Flores L, Cedeño R, y Rentería Luis. (2018). Acceso a las Tic`s en estudiantes Afroecuatorianos en Santo Domingo de los Tsáchilas. Revista electrónica Ciencia Digital 2(1), 161-175. Recuperado desde:

http://www.cienciadigital.org/revistascienciadigital2/index.php/CienciaDigital/article/view/ $11 / 11$

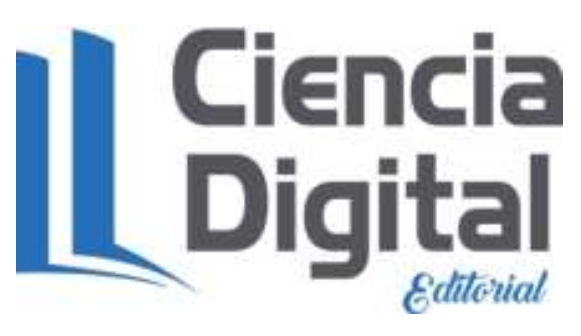

El artículo que se publica es de exclusiva responsabilidad de los autores y no necesariamente reflejan el pensamiento de la Revista Ciencia Digital.

El articulo queda en propiedad de la revista y, por tanto, su publicación parcial y/o total en otro medio tiene que ser autorizado por el director de la Revista Ciencia Digital.
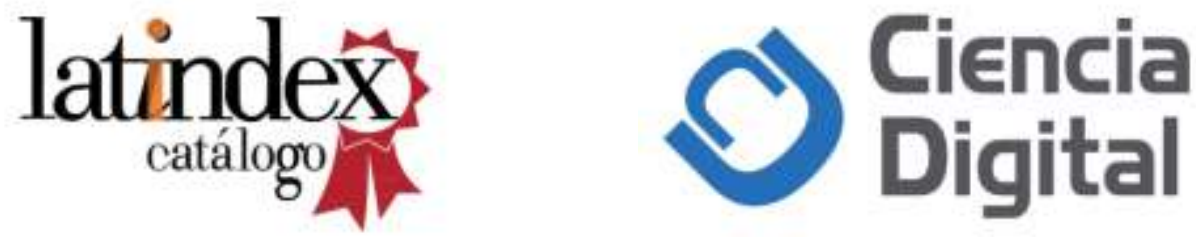